\title{
An "Old" Approach to the Damage of the Peripheral Nervous System: Do we Still Need Morphology?
}

\section{Guido Cavaletti ${ }^{\star}$ and Paola Marmiroli}

Department of Neuroscience and Biomedical Technology, University of Milano-Bicocca, Monza, Via Cadore 48, 20052 Monza (MB), Italy

Research improvement in the field of neuroscience greatly profited from the use of modern techniques based on high-resolution noninvasive imaging, neurophysiological investigation, biomarkers analysis and behavioral assessment. Given the huge amount of sophisticated and highly technological tools available in our laboratories, researchers might find rather obsolete the use of morphological investigation and they can consider training in morphological sciences as a low-return activity. In fact, the observation of biological sample is frequently perceived as an "old", time consuming, investigator-dependent, highlyvariable approach to experimental neuroscience, hardly able to really capture the normal, physiologic condition that turned to be pathologic in animal models of human diseases. However, this perception is definitely wrong and several examples could be offered in support of the still fundamental importance of the morphological examination of the nervous system and of the need for an extensive training in this technique.

These statements are based on our personal experience as investigators and tutors for young researchers in the $\mathrm{PhD}$ program in Neuroscience at our University, where the morphology laboratory is placed at the center of any experimental activity. Although several different examples might be provided to support the fundamental role of the morphological investigation, the study of the peripheral nervous system (where for instance non-invasive imaging is still poorly informative) gives one of the most clear evidence of its relevance and we can give the example provided by the study of chemotherapyinduced peripheral neurotoxicity (CIPN) [1].

CIPN is still a highly-relevant, umnet clinical need since it can be severe, with a remarkable impact on the quality of life of cancer patients and it is a potentially dose limiting side effect of the modern antineoplastic chemotherapy [2]. Its investigation is mandatory since no treatment is currently available to prevent its onset and clinical sign may be long-lasting or even permanent. The peripheral nervous system is a frequent target of the neurotoxicity of several anticancer compounds and the accurate identification of their sites of neurotoxic action has provided valuable clues to understand the pathogenesis and interpreting the clinical features of CIPN.

In fact, while the clinical and neurophysiological features of CIPN may only suggest the possible target of toxicity, morphological studies performed on human specimens (when available) or using reliable animal models can actually demonstrate if a structure is really involved in the pathological process and to what extent. As a further evidence, although the clinical aspects of CIPN induced by different drugs might suggest a common pathogenesis, specific morphological features of neuronal and glial cells damage induced at different levels by platinum compounds, taxanes, vinca alkaloids and bortezomib have been demonstrated, clearly indicating that these drugs act with different mechanisms and on different intracellular targets [3].
These studies have also pointed out that animal models are fundamental in the study of CIPN, since the availability of tissues of human origin is extremely limited. In fact, most of the neurotoxic drugs have some pathologic effect on the dorsal root ganglia neurons, and it is a common experience among morphologists that it is very difficult to obtain high-quality human specimens even from early autopsies.

Supporting the importance of the morphological assessment does not imply that other types of investigation (e.g. neurophysiological and behavioral studies) should be discarded or under-valued in their contribution, but rather than only the complementarity of all the available techniques might really allow a sound scientific development of the modern neuroscience, although the morphological examination still remains the "golden standard" for most studies.

We are therefore convinced that, even in early $21^{\text {st }}$ century, the amount, the quality and the relevance of the information provided by a focused morphological assessment, possibly supported by a morphometric analysis must be considered fundamental research tools and that efforts should be continuous to train our younger fellows in this difficult methodology which tends to be frequently underestimated in its importance.

\section{References}

1. Cavaletti G (2009) Chemotherapy-induced peripheral neurotoxicity: how can we improve knowledge? Lancet Oncol 10: 539-540.

2. Cavaletti G, Marmiroli P (2010) Chemotherapy-induced peripheral neurotoxicity Nat Rev Neurol 6: 657-666.

3. Cavaletti G, Nicolini G, Marmiroli P (2008) Neurotoxic effects of antineoplastic drugs: the lesson of pre-clinical studies. Front Biosci 13: 3506-3524.

${ }^{*}$ Corresponding author: Prof. Guido Cavaletti, Department of Neuroscience and Biomedical Technology, University of Milano-Bicocca, Monza, Via Cadore 48, 20052 Monza (MB), Italy, Tel: +39-02-6448 8039; Fax: +39 026448 8250; E-mail: guido.cavaletti@unimib.it

Received July 17, 2012; Accepted July 18, 2012; Published July 20, 2012

Citation: Cavaletti G, Marmiroli P (2012) An "Old" Approach to the Damage of the Peripheral Nervous System: Do we Still Need Morphology? J Cytol Histol 3:e105. doi:10.4172/2157-7099.1000e105

Copyright: (c) 2012 Cavaletti G, et al. This is an open-access article distributed under the terms of the Creative Commons Attribution License, which permits unrestricted use, distribution, and reproduction in any medium, provided the original author and source are credited. 